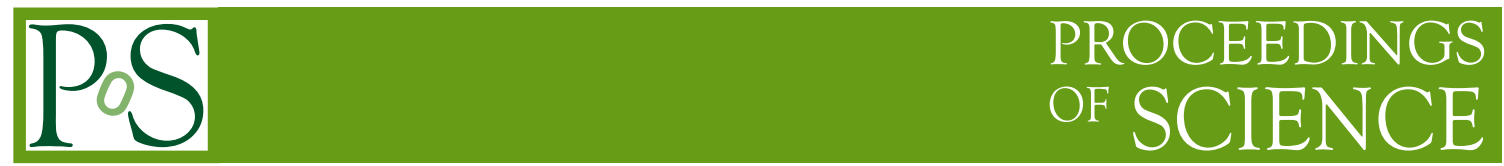

\title{
LHCb: Recent Results and Future Plans
}

\section{C.L. da Silva on behalf of the LHCb Collaboration ${ }^{a, *}$}

${ }^{a}$ Los Alamos National Lab,

Physics Division, Los Alamos/NM, USA

E-mail: cesar_luiz@lanl.gov

In this overview contribution we introduce the most recent results in high-multiplicity $p p$ and heavy ion collisions obtained by the LHCb experiment, detector developments and plans for the future heavy ion program. Some of the highlights include the study of double charm production, nuclear modification of $\mathrm{B}$-hadrons, quarkonia and $\mathrm{Z}$-bosons in $\mathrm{pPb}$ and the suppression of prompt $\mathrm{X}(3872)$ hadrons in high multiplicity $p p$ events. On the detector side, we bring to attention the preparation for the upcoming Run III in LHC and the fixed target program.

HardProbes 2020

1-6 June 2020

Austin, Texas

${ }^{*}$ Speaker 


\section{The LHCb detector and heavy ion data sets.}
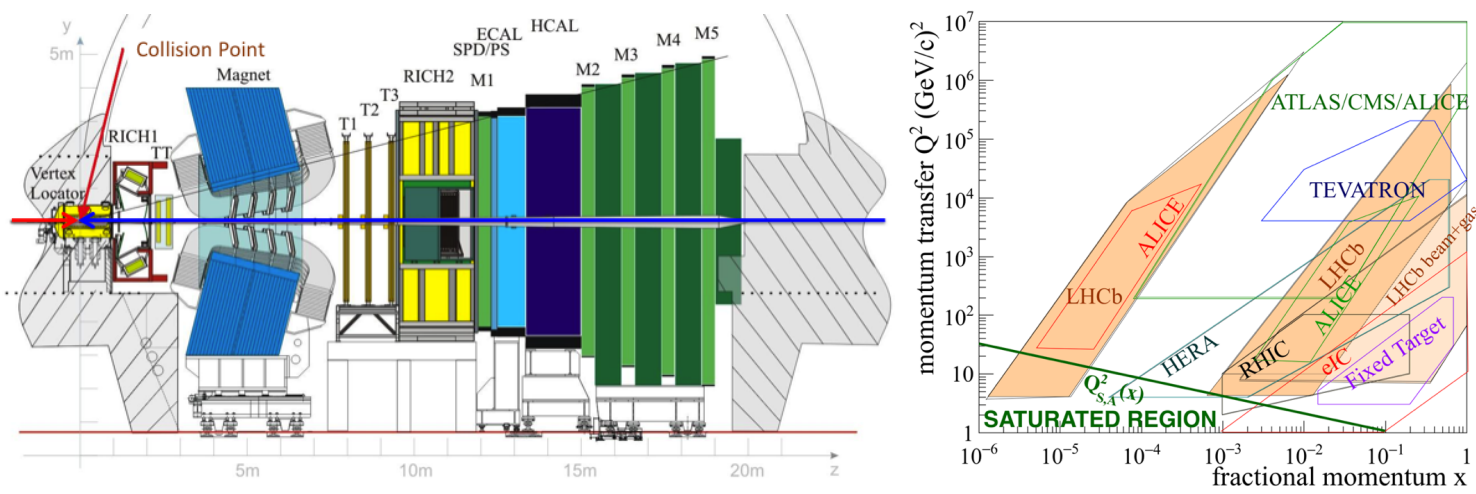

Figure 1: (left) The LHCb apparatus during Run I and II. (right) Parton kinematic coverage of past, recent and future facilities with emphasis on the expected gluon saturated regime based on estimations made in [1].

The Large Hadron Collider beauty experiment (LHCb) [2] is a general purpose single arm detector covering the pseudorapidity region of $1.6<\eta<4.9$ (Fig. 1-left) with $e, \mu, \pi, \mathrm{K}, p$ and $\gamma$ identification in a momentum ranging in $1-100 \mathrm{GeV} / c$. The detector has jet reconstruction capabilities and interaction point resolution $<80 \mu \mathrm{m}$. In the Run2 configuration, which just ended, the data acquisition can reach rates of $1 \mathrm{MHz}$. These capabilities makes LHCb the detector with the best instrumentation covering the forward region among the LHC experiments. The combination of the high energy collisions of LHC and its forward coverage makes LHCb a unique detector for partonic studies in a broad kinematic range, as seen in Figure 1-right.

LHCb collected data in $p \mathrm{~Pb}, \mathrm{~Pb} p$ at $\sqrt{s_{N N}}=5.02 \mathrm{TeV}$ and $\sqrt{s_{N N}}=8.16 \mathrm{TeV}$ and $\mathrm{PbPb}$ collisions at $\sqrt{s_{N N}}=5.02 \mathrm{TeV}$ in the standard beam-beam collisions mode of LHC. The recorded luminosity tops around $10 \mathrm{nb}^{-1}$ in $p \mathrm{~Pb} / \mathrm{Pbp}$ collisions and $0.2 \mathrm{nb}^{-1}$ in $\mathrm{PbPb}$ collisions. The data acquisition is limited to the $40 \%$ most peripheral events in $\mathrm{PbPb}$ collisions because of the occupancy saturation in the inner vertex detector (VELO). The fixed target mode, using beam-gas collisions inside the VELO detector, started as a pilot in 2015 and now is integrated to the LHCb Physics program. The center of the mass collision energy ranges from $69 \mathrm{GeV}$ to $115 \mathrm{GeV}$ in beam-gas collisions. A summary of the species, beam energies and recorded luminosity is shown in Figure 2.
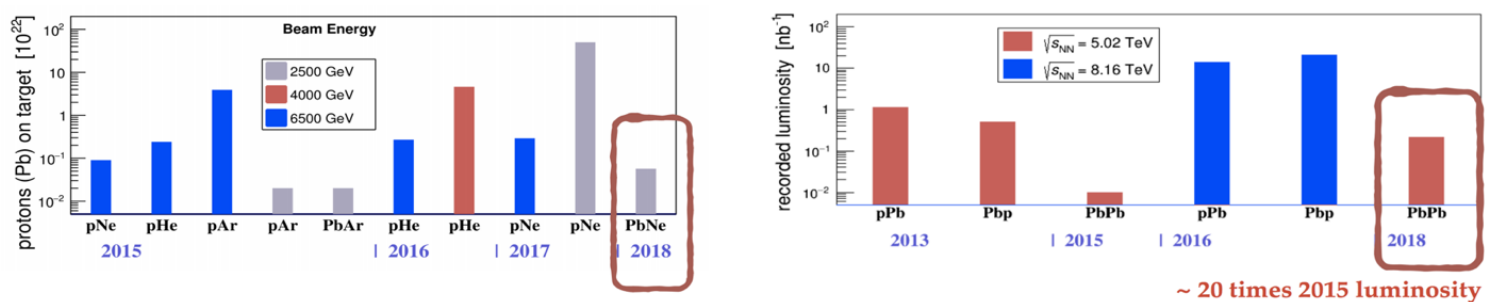

Figure 2: Heavy ion data sets collected by the LHCb experiment in fixed target mode (left) and beam-beam collisions (right). 


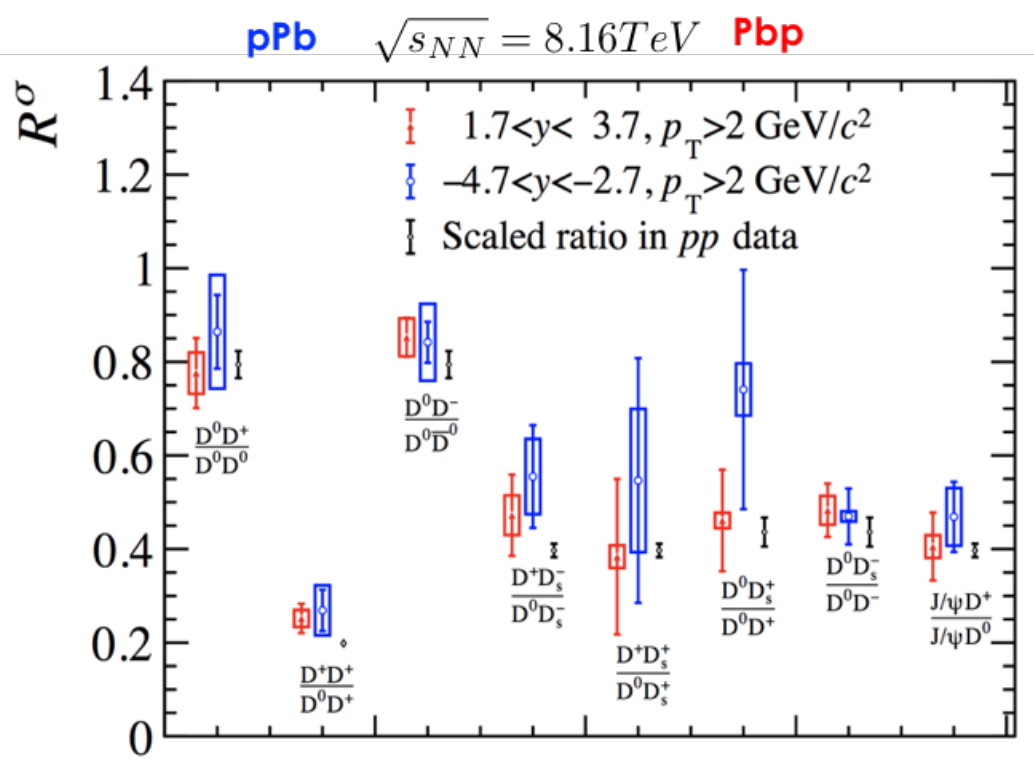

Figure 3: Yield ratio between different charm hadron pairs measured in $p \mathrm{~Pb}$ and $\mathrm{Pb} p$ collisions at $\sqrt{s_{N N}}=8.16 \mathrm{TeV}$ along with $p p$ ratios obtained from sngle hadron cross-sections scaled by the number of charm-quark hadronization paths into charm pairs.

\section{Recent Physics results.}

The study of double charm production started in $p p$ collisions in the early days of the LHCb operation [3] using pairs of several combinations of charm hadrons and $J / \psi$. The analysis found the pair cross-sections and angular correlations indicate large contribution of $c \bar{c}$ produced in gluon splitting processes in single parton scattering (SPS) processes and double parton scattering (DPS) [4]. The existence of DPS is evident in like-sign pairs (LS) and reveals the level of parton correlation in the proton, implemented in the cross-section calculations as $\sigma_{e f f}$. This study was extended to $p \mathrm{~Pb}$ collisions showing an enhancement of LS production by an average factor of three in $p \mathrm{~Pb}$ collisions (positive rapidity) and even larger in $\mathrm{Pb} p$ collisions (negative rapidity) [5]. The LS enhancement and the angular distributions indicates the enhancement of DPS processes in $\mathrm{pPb}$, in agreement with expected geometric effects in such collisions predicted in [6]. The yield ratio between different combinations of opposite-sign charm hadron pairs cross-sections from SPS processes, shown in Figure 3, indicates agreement with what is expected from $p p$ cross-sections and the number of charm-quark hadronization paths into charm pairs. This observation suggests there is no significant modification of charm hadronization in $p \mathrm{~Pb}$ and $\mathrm{Pb} p$ collisions compared to $p p$ collisions. The contribution [7] contains more information on the double charm production measurements performed by the LHCb.

Heavy flavor production in $p \mathrm{~A}$ collisions is one of the well known probes for initial-state nuclear effects and nuclear Parton Density Function (nPDF) constraints. LHCb has measured Dhadrons [8], B-hadrons [9] and non-prompt $\mathrm{J} / \psi$ [10] in forward and backward rapidity spanning its coverage between $5.0<y^{<} 2.5$ and $1.5<y^{<} 4.0$, where $y^{*}$ is the rapidity at center of the mass of the colliding nucleons. The LHCb data are typically more precise than the current nPDFs 
and it has been used to additionally constrain the EPPS16 nPDF[11, 12]. The most recent result from $\mathrm{B}^{+}$nuclear modification in $p \mathrm{~Pb}$ collisions at $\sqrt{s_{N N}}=8.16 \mathrm{TeV}$, published in [9], shows agreement with previous measurements using non-prompt $\mathrm{J} / \psi$ and nPDFs. It is noteworthy the estimation for B-meson nuclear modification using the LHCb data constrained EPPS16* [11] is also in agreement with the data, indicating the universality of the nPDF. The same publication also reports no significant modification of the beauty baryon/meson ratio in small systems. A tendency of modification of this ratio is observed at backward rapidity but the current uncertainties provides no conclusions. More details on the recent open heavy flavor results from LHCb can be found in [7].

The Z-boson measurement in $p \mathrm{~Pb}$ and $\mathrm{Pb} p$ collisions at $\sqrt{s_{N N}}=8.16 \mathrm{TeV}$ [13] also provides a strong constraining to nPDF at high virtuality $Q^{2}$ scales and parton fractional momentum $x \sim 10^{-3}$ in $p \mathrm{~Pb}$ and $x \sim 0.3$ in $\mathrm{Pb} p$. The results shows a tension with the nCTEQ15 nPDF [14] in both forward and backward rapidities, despite its success in describing the data obtained at mid-rapidity by ATLAS [15] and CMS [16]. Other PDFs, such as NNPDF31 [17] and its combination with EPPS16 [18], provides a better agreement with the LHCb data at forward rapidity with some tension at backward rapidity (large- $x$ ) region. The contribution [19] to this volume provides more details on the Z-boson measurements at LHCb.

Being an experiment designed for flavor Physics measurements, $\mathrm{LHCb}$ is well positioned to expand the number of quarkonia states measured in collisions involving nucleus. One example is the first observation of $\chi_{c 1,2}$ peaks in $p \mathrm{~Pb}$ and $\mathrm{Pb} p$ collisions [20]. The detector is also able to measure $\mathrm{J} / \psi, \psi(2 \mathrm{~S})$ and separated $\Upsilon(1 \mathrm{~S}, 2 \mathrm{~S}, 3 \mathrm{~S})$ from zero $p_{T}$ along with $\mathrm{D}^{0}$ mesons. This capability allows LHCb to study quarkonia breaking/melting in heavy ion collisions and isolate initial-state effects which are common for quarkonia and heavy flavor probes. The paper [8] reporting D-meson production shows $\mathrm{J} / \psi$ and $\psi(2 \mathrm{~S})$ nuclear modifications relative to $\mathrm{D}^{0}$ at forward and backward rapidity indicating no significant suppression of prompt $\mathrm{J} / \psi$ relative to prompt $\mathrm{D}^{0}$. On the other hand, $\psi(2 \mathrm{~S})$ indicates a suppression larger than D-mesons, especially at the backward rapidity. Similar, initial-state effect free, measurements were performed with $\Upsilon$ states in [21], using the ground state $\Upsilon(1 \mathrm{~S})$ as a reference. LHC is also unique in measuring quarkonia in fixed target mode, such as the $\mathrm{J} / \psi$ peak obtained in PbNe collisions at $\sqrt{s_{N N}}=69 \mathrm{GeV}$ during the 2018 run. The contribution [22] describe the most recent quarkonia results and plans from LHCb.

During this conference we are witnessing the surge of a new line of high-energy Physics research: the identification of particle size and structure by using comoving particle interactions. Since its discovery in the early 2000s, X(3872) is known to be a particle composed of two valence charm and two light quarks but with a disputed description of its size and structure. This charmed hadron could be a tightly bound tetraquark or a loosely bound $D^{0} \bar{D}^{*}$ molecule system. The event track multiplicity dependency of $\psi(2 \mathrm{~S})$ and $\mathrm{X}(3872)$ yields were measured in $p p$ collisions at $\sqrt{s}=8$ $\mathrm{TeV}$ [24]. The relative ratio of these yields indicate a suppression of prompt $\mathrm{X}$ (3872) relative to $\psi(2 S)$ in high multiplicity events. This relative suppression indicates the $\mathrm{X}(3872)$ breaks more than $\psi(2 \mathrm{~S})$ when interacting with comoving particles. The LHCb measurement was compared to a calculation involving comoving interactions with the $\mathrm{X}(3872)$ hypothesis of a molecular particle or a tightly bound tetraquark [23]. These models were successful in describing quarkonia suppression in small and large nuclear systems. Figure 4-left shows the data strongly supports the observation of tightly bound tetraquark $\mathrm{X}(3872)$ with $1.3 \mathrm{fm}$ size being suppressed by the comoving particles 

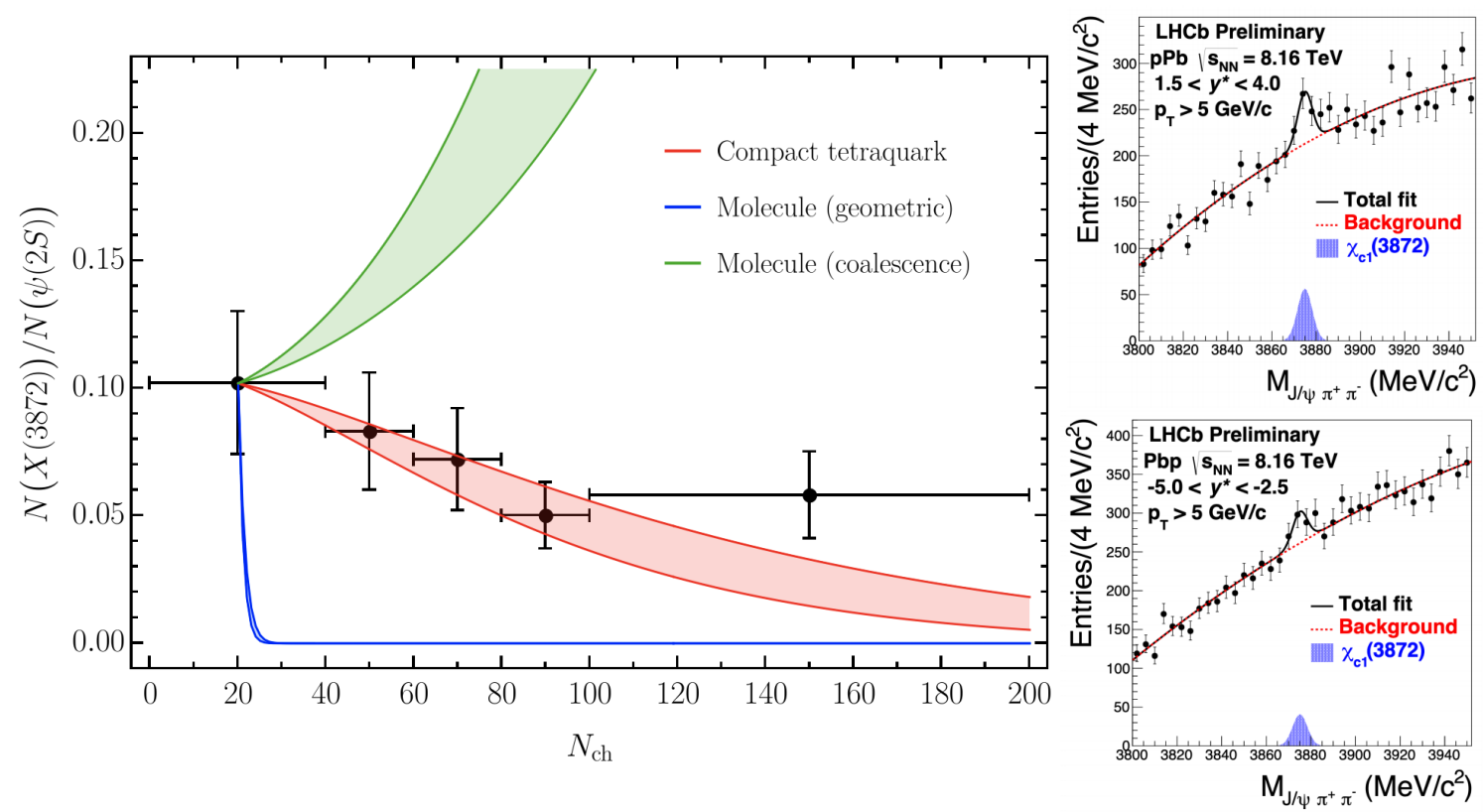

Figure 4: (left)Track multiplicity dependency of prompt X(3872) yield suppression relative to prmpt $\psi(2 \mathrm{~S})$ yield observed in $p p$ collisions along with prediction for different hypothesis for the nature of the $\mathrm{X}(3872)$ [23]. (right) $\mathrm{X}(3872)$ peaks observed in $p \mathrm{~Pb}$ and $\mathrm{Pb} p$ collisions.

produced in $p p$ collisions. According to the model, $D^{0} \bar{D}^{*}$ molecules would be broken even in events with small activity. The scenario where $\mathrm{X}(3872)$ is produced by coalescence is also not supported by the data. Future results from the $\mathrm{X}(3872)$ peaks observed in $p \mathrm{~Pb}$ and $\mathrm{Pb} p$ collisions (Fig. 4-right) will allow the reach of a larger event track mutiplicity revealing any possible modification of the behavior of X(3872) in nuclear medium.

\section{Future Plans.}

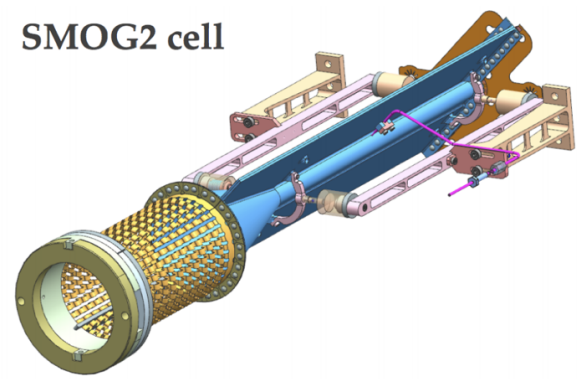

Figure 5: Illustration of the new SMOG target cell dedicated to beam-gas collisions [25].

The LHCb detector is going through a near complete upgrade during the LHC Long Shutdown II [26]. The upgrades include a new inner vertex detector (VELO) with pixel segmentation, new RICH PMTs and electronics, new tracking stations. All the readout electronics are being replaced to provide a software-only trigger system with $40 \mathrm{MHz}$ data acquisition and real time data 
reconstruction. The LHCb is innovating in being the first high-energy Physics experiment with real time analysis. The new detector configuration will reduce the occupancy limitation in $\mathrm{PbPb}$ collisions allowing to collect data over the $70 \%$ most peripheral events. The remaining hardware limitation will be caused by the scintillating fiber based tracker ( $\mathrm{SiFi})$.

The most important upgrade concerning the heavy ion program is the introduction of the SMOG2 cell (Fig. 5) for beam-gas fixed target collisions [25]. The new target cell will have 100 times higher gas pressure compared to the current SMOG, allowing orders of magnitude higher number of beam+gas collisions. The luminosity uncertainties are expected to be smaller than $6 \%$, the LHCb fixed target mode has no centrality limitations and it can run parasitically during $p p$ runs. Yields of 150 million $D^{0}$ mesons, or $7 \mathrm{~K} \Upsilon(1 \mathrm{~S})$ yields will be observed in one run year operation in $p$ Ar collisions at $\sqrt{s_{N N}}=115 \mathrm{GeV}$. This future heavy ion program will cover an intermediate collision energy between SPS and RHIC, greatly expanding the understanding of quark-gluon plasma formation and composition.

The contribution [27] to this edition is dedicated to describe the LHCb upgrades and its future heavy ion program.

\section{Conclusions.}

The experimental results from the LHCb heavy ion program is already providing essential information for the understanding of hard processes in heavy ion collisions. Nuclear parton distributions are greatly constrained by LHCb results with open heavy flavor, Z-bosons and quarkonia. New results on double charm production reveals the importance of parton correlations in nuclear collisions. LHCb presented the first results where comoving particles are used to understand exotic particle composition and size. Other exotic particles may come, thanks to the excellent particle identification and vertexing of LHCb. There are several results in the pipilene, such as quarkonia and heavy flavor in fixed target collisions, new quarkonia states like $\chi_{c}$, gluon saturation search with direct photons, vector mesons in ultra-peripheral collisions, and others. Many more analysis are waiting for analyzers.

The $\mathrm{LHCb}$ has a bright future after the long-shutdown II with reduced limitations in $\mathrm{PbPb}$ collisions and a high luminosity fixed target program. It is a very nice opportunity to explore new territories in high-energy nuclear collisions with this unique experimental apparatus.

\section{References}

[1] H. Kowalski, T. Lappi, and R. Venugopalan. Nuclear enhancement of universal dynamics of high parton densities. Phys. Rev. Lett., 100:022303, 2008.

[2] The LHCb Collaboration. The lhcb detector at the lhc. Journal of Instrumentation, 3(08):S08005, 2008.

[3] R Aaij et al. Observation of double charm production involving open charm in pp collisions at $\sqrt{s}=7 \mathrm{TeV}$. JHEP, 06:141, 2012. [Addendum: JHEP 03, 108 (2014)].

[4] Rafał Maciuła and Antoni Szczurek. Double-parton scattering effects in associated production of charm mesons and dijets at the lhc. Phys. Rev. D, 96:074013, Oct 2017. 
[5] Roel Aaij et al. Observation of enhanced double parton scattering in proton-lead collisions at $\sqrt{s_{\mathrm{NN}}}=8.16 \mathrm{TeV} .72020$.

[6] David d'Enterria and Alexander M. Snigirev. Same-sign WW production in proton-nucleus collisions at the LHC as a signal for double parton scattering. Phys. Lett. B, 718:1395-1400, 2013.

[7] Jianqiao Wang. Open heavy flavor production in pa collisions. Hard Probes 2020 proceedings in this volume.

[8] Roel Aaij et al. Study of prompt $\mathrm{D}^{0}$ meson production in $p \mathrm{~Pb}$ collisions at $\sqrt{s_{\mathrm{NN}}}=5 \mathrm{TeV}$. JHEP, 10:090, 2017.

[9] Roel Aaij et al. Measurement of $B^{+}, B^{0}$ and $\Lambda_{b}^{0}$ production in $p \mathrm{~Pb}$ collisions at $\sqrt{s_{\mathrm{NN}}}=$ 8.16 TeV. Phys. Rev. D, 99(5):052011, 2019.

[10] R. Aaij et al. Prompt and nonprompt $\mathrm{J} / \psi$ production and nuclear modification in $p \mathrm{~Pb}$ collisions at $\sqrt{s_{\mathrm{NN}}}=8.16 \mathrm{TeV}$. Phys. Lett. B, 774:159-178, 2017.

[11] Aleksander Kusina, Jean-Philippe Lansberg, Ingo Schienbein, and Hua-Sheng Shao. Gluon Shadowing in Heavy-Flavor Production at the LHC. Phys. Rev. Lett., 121(5):052004, 2018.

[12] Kari J. Eskola, Ilkka Helenius, Petja Paakkinen, and Hannu Paukkunen. A QCD analysis of LHCb D-meson data in p+Pb collisions. JHEP, 05:037, 2020.

[13] Hengne Li. Measurement of $\mathrm{Z}$ production cross-section in proton-lead collisions at $\sqrt{\mathrm{sNN}}=$ $8.16 \mathrm{TeV} .112019$.

[14] K. Kovarik et al. nCTEQ15 - Global analysis of nuclear parton distributions with uncertainties in the CTEQ framework. Phys. Rev. D, 93(8):085037, 2016.

[15] Georges Aad et al. $Z$ boson production in $p+\mathrm{Pb}$ collisions at $\sqrt{s_{N N}}=5.02 \mathrm{TeV}$ measured with the ATLAS detector. Phys. Rev. C, 92(4):044915, 2015.

[16] Vardan Khachatryan et al. Study of $\mathrm{Z}$ boson production in $\mathrm{pPb}$ collisions at $\sqrt{s_{N N}}=5.02$ TeV. Phys. Lett. B, 759:36-57, 2016.

[17] Richard D. Ball et al. Parton distributions from high-precision collider data. Eur. Phys. J. C, 77(10):663, 2017.

[18] Sayipjamal Dulat, Tie-Jiun Hou, Jun Gao, Marco Guzzi, Joey Huston, Pavel Nadolsky, Jon Pumplin, Carl Schmidt, Daniel Stump, and C.P. Yuan. New parton distribution functions from a global analysis of quantum chromodynamics. Phys. Rev. D, 93(3):033006, 2016.

[19] Hengne Li. Z production in collisions at lhcb. Hard Probes 2020 proceedings in this volume.

[20] $X\rfloor \infty(3872)$ production in $p \mathrm{~Pb}$ and $\mathrm{Pb} p$ collisions at $\sqrt{s N N}=8.16 \mathrm{TeV}$. Oct 2019 .

[21] Roel Aaij et al. Study of $\Upsilon$ production in $p$ Pb collisions at $\sqrt{s_{N N}}=8.16 \mathrm{TeV}$. JHEP, 11:194, 2018. [Erratum: JHEP 02, 093 (2020)]. 
[22] Oscar Boente Garcia. Quarkonia production in ppb collisions. Hard Probes 2020 proceedings in this volume.

[23] Angelo Esposito, Elena G. Ferreiro, Alessandro Pilloni, Antonio D. Polosa, and Carlos A. Salgado. The nature of $X(3872)$ from high-multiplicity $p p$ collisions. 62020.

[24] Multiplicity-dependent modification of $\chi_{c 1}(3872)$ and $\psi(2 S)$ production in $p p$ collisions at $\sqrt{s}=8 \mathrm{TeV}$. Technical Report LHCb-CONF-2019-005. CERN-LHCb-CONF-2019-005, CERN, Geneva, Nov 2019.

[25] Albert Bursche et al. Physics opportunities with the fixed-target program of the LHCb experiment using an unpolarized gas target. Technical Report LHCb-PUB-2018-015. CERNLHCb-PUB-2018-015, CERN, Geneva, Dec 2018.

[26] I. Bediaga et al. Framework TDR for the LHCb Upgrade: Technical Design Report. 42012.

[27] Samuel Belin. Lhc run3 and run4 prospects for heavy-ion physics with lhcb. Hard Probes 2020 proceedings in this volume. 